\title{
RAPID DISASTER DAMAGE ESTIMATION
}

\author{
T. T. $\mathrm{Vu}^{\mathrm{a}}$ \\ ${ }^{\text {a }}$ School of Geography, University of Nottingham, Malaysia campus, Jalan Broga, Semenyih, 43500 Selangor, \\ Malaysia - tuongthuy.vu@nottingham.edu.my
}

Commission VIII, WG VIII/1

KEY WORDS: Remote Sensing, Feature Extraction, Segmentation, Disaster, Damage mapping

\begin{abstract}
:
The experiences from recent disaster events showed that detailed information derived from high-resolution satellite images could accommodate the requirements from damage analysts and disaster management practitioners. Richer information contained in such high-resolution images, however, increases the complexity of image analysis. As a result, few image analysis solutions can be practically used under time pressure in the context of post-disaster and emergency responses. To fill the gap in employment of remote sensing in disaster response, this research develops a rapid high-resolution satellite mapping solution built upon a dual-scale contextual framework to support damage estimation after a catastrophe. The target objects are building (or building blocks) and their condition. On the coarse processing level, statistical region merging deployed to group pixels into a number of coarse clusters. Based on majority rule of vegetation index, water and shadow index, it is possible to eliminate the irrelevant clusters. The remaining clusters likely consist of building structures and others. On the fine processing level details, within each considering clusters, smaller objects are formed using morphological analysis. Numerous indicators including spectral, textural and shape indices are computed to be used in a rule-based object classification. Computation time of raster-based analysis highly depends on the image size or number of processed pixels in order words. Breaking into 2 level processing helps to reduce the processed number of pixels and the redundancy of processing irrelevant information. In addition, it allows a data- and tasks- based parallel implementation. The performance is demonstrated with QuickBird images captured a disaster-affected area of Phanga, Thailand by the 2004 Indian Ocean tsunami are used for demonstration of the performance. The developed solution will be implemented in different platforms as well as a web processing service for operational uses.
\end{abstract}

\section{INTRODUCTION}

Providing quick and reliable captured information in hardest hit and difficult-to-assess areas, remote sensing products have been commonly used as the first and primary information source at the post-disaster response stage (Adams et al., 2004; Balz and Liao, 2010; Matsuoka and Yamazaki 1999; Saito et al., 2004; Stramondo et al., 2006; Vu et al. 2005). The activation of International Charter on Space and Major Disasters (www.disastercharter.org) together with the coordination of UN-SPIDER (www.un-spider.org) facilitates the acquiring and delivering timely remote sensing images to the bodies in charge of relief efforts and emergency responses. The fastest available information is damage extent that is manually extracted in such operational framework. The product accuracy with respect to the practices is far to meet (Kerle 2010). More quantitative and details of damages are expected from the damage analysts and disaster management practitioners. Researches in response to recent disaster events showed that detailed information derived from very high-resolution satellite images could accommodate their requirements (Chesnel et al. 2007; Gusella et al. 2005; Saito et al. 2004; Vu and Ban, 2010).

Richer information contained in such high-resolution images increases the complexity of image analysis. Numerous researches have been done either to develop or employ the object-based image analysis approach (Blaschke 2010), which proved to be the most suitable for high-resolution satellite images. Those, however, are impractically used under time pressure in the context of post-disaster and emergency responses due to the high computational cost and the requirement of experienced operators. Since no mature automated image analysis solution is available, the practitioners are seeking the collaborative mapping framework (Goodchild and Glennon 2010). GEO-CAN (Bevington et al. 2010), a practical work has been efficiently deployed in response to the 2010 Haiti earthquake, in which the huge time-consuming interpretation of damages from remote sensing images were divided into gird-based delegation to numerous contributors worldwide. As collaborative mapping platform is well developed, the mechanism to ensure the accuracy and consistency is the big challenge. Moreover, damage interpretation requires a certain level of expertise in remote sensing and structural engineering. The quick image processing outcomes would be a guideline for the contributors as well as a reference frame to ensure the quality of derived damage information.

To contribute to the current efforts, this research develops a rapid high-resolution satellite mapping solution built upon a dual-scale contextual framework to support damage estimation after a catastrophe. The initial development is formulated as a part of a tsunami-damage estimation system (Koshimura et al. 2010) integrating numerical modelling of tsunami inundation, remote sensing and GIS. High-resolution remote sensing images are acquired to update the surface roughness for tsunami modelling as well as mapping the structural damages. Thus, the target objects of image processing are building (or building blocks). Breaking into two levels of processing, the design and implementation are optimized for computation speed. Details of developed solutions are described in Section 2 and demonstrated with QuickBird images of Ban Nam Ken, Phanga, Thailand in Section 3. 


\section{METHODOLOGY}

Image- and/or raster-based computation time heavily depends on image size. To cover a considerably large area, the size of a QuickBird, IKONOS, or WorldView-2 image becomes huge multiplied by its multi-spectral bands. On another hand, objectbased image analysis exploits the scale property of objects in describing the context for segmentation and classification. The dual-scale processing proposed here takes into account the scale property not only categorise objects into different levels for segmentation and classification but also to decide the suitability level of complexity in processing in order to shorten the computation time.

The two levels of the main processing are described in the following sub-sections. It is noted that pre-processing such as geometric and radiometric correction, pan-sharpening may be necessary prior to the main processing. Those are easily done by any remote sensing packages and not repeated here.

\subsection{Coarse level}

On the coarse level, the simple but robust Statistical Region Merging (SRM) technique is adopted (Nock and Nielsen, 2004). SRM starts by sorting the pair of pixels in ascending order of $f\left(p, p^{\prime}\right)$, expressed as in Eq. 1.

$$
f\left(p, p^{\prime}\right)=\max _{\forall a \in\{S\}}\left|p_{a}-p_{a^{\prime}}\right|,
$$

$a$ denotes a spectral channel of the multi-spectral space $S$

Traversing the above order once and testing for any pair, if the region $R$ and $R^{\prime}$ of the pair are different, $R$ and $R^{\prime}$ will be merged if

$$
\left|\bar{R}_{a}^{\prime}-\bar{R}_{a}\right| \leq \sqrt{b^{2}(R)+b^{2}\left(R^{\prime}\right), \forall} \forall \in\{S\},
$$

where $\bar{R}$ denotes the average value of region $R$

$b(R)=g \sqrt{\frac{1}{2 Q|R|}\left(\ln \frac{\left|\Re_{l}\right|}{\delta}\right)}$, with $\left|\Re_{l}\right|=(l+1)^{\min (l, g)}$,

$g$ denotes the number of grey value,

$Q$ is tuning parameter, $\delta=\frac{1}{3|I|^{2}}$,

$|I|$ and $|R|$ denote entire image and region $R$ size, respectively.

More details of SRM are proved and presented in Nock and Nielsen's papers (Nock and Nielsen 2004, Nock and Nielsen 2005). To delineate the big objects, the $Q$ value is fixed at 64 whereas the $g$ value is fixed to 256 so it is required to scale the simplified image grey value to $0-255$. SRM is conventionally working on pixels that may take long time. To assist speeding up the conditional search based upon Eq. 2, the initial clusters are generated via K-mean clustering and morphological filtering (Vincent 1993). The filtering is to remove the small meaningless objects, less than 25 pixels, and is applied onto the spectral clustered images derived by K-mean. K-mean clustering helps to initially group the pixels using their multispectral signatures globally. The locally grouping is then controlled by SRM. After filtering, the pixels in a neighbour that have similar grey value (class number) will be merged into a cluster. Those clusters will play as the basic entity for SRM instead of pixels.

Subsequently, the rule-based analysis is carried out to decide which objects should be go further to fine level processing as well as remove irrelevant objects such as water and vegetation.
First, homogeneous texture is measured, those with low homogeneous value is subject to go further. To discriminate water, vegetation from impervious surface, the brightness and greenness indices (Yarbrough et al. 2005) in combination with NDVI are employed. High level of greenness indicates the vegetation cover after confirmed with high NDVI value, whereas both low brightness and greenness values indicate the water body. The impervious surfaces have low greenness and high brightness values. The outcomes of coarse level processing, therefore, include the following types: homogeneous irrelevant objects such as water and vegetation, homogenous big impervious surfaces objects, and heterogeneous objects to go further to fine level processing. The very low homogeneous objects with low brightness and greenness values would be a cue to focus in mapping the damages. The whole process is illustrated in Figure 1.

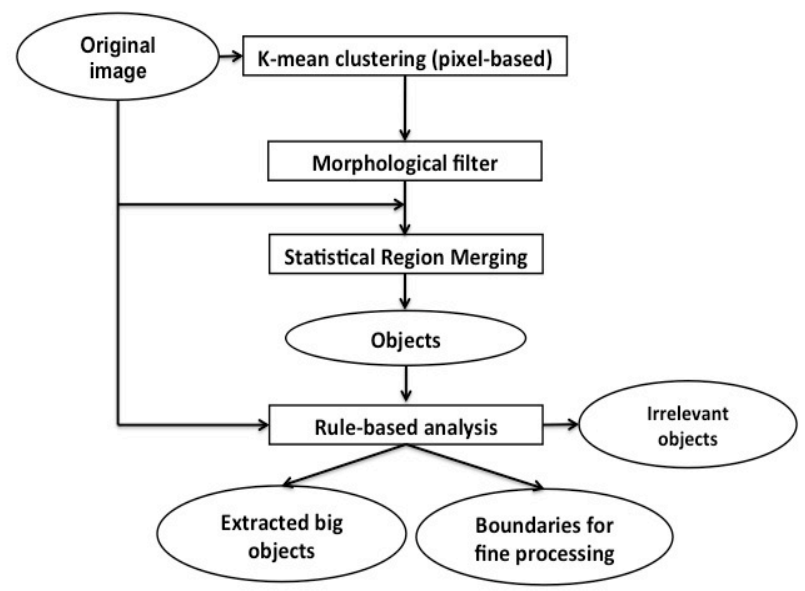

Figure 1. Coarse level processing

\subsection{Fine level}

In the detailed processing, a considerably large area has been masked out on coarse level with expectation that the time processing will be shortened. The flowchart of this fine level processing is shown in Figure 2. Going into details, it is possible to reveal the geometrical beside the spectral properties of each object. The previously developed non-linear scale space transformation by the author ( Vu and Ban 2010) is adopted here to derive the morphological profile and form the fine objects. Again, to speed up the processing, morphological profile is generated on only the first component of PCA.

Briefly, morphological filtering with reconstruction with increasing template size is applied onto the first component analysis. The morphological profiles across the scale space provide the cues to group nearby pixels to a cluster via similarity measurement. It also helps to group the clusters with similarity profile to the same class. Since it works with only small objects, a limited number of sizes of increasing step of 1 can be used to generate the scale-space, named the scale range. Granulometry analysis is also employed to ignore the unnecessary sizes at which no significant changes on the granulometry spectrum within the scale range.

In addition, the compactness and elongated shape indices (Bogaert et al. 2000), ratio between object area and object perimeter/length, are exploited to discriminate building and road objects. The rest of spectral information is added back via a simple K-mean pixel-based classification. The spectral class of 
a fine object is decided based on the majority rule of pixelbased spectral classes within it.

New set of rule is established with regards to fine objects and their newly derived properties. As the potential damage area can be delineated from coarse level processing, this fine level processing mainly focuses in detection of intact building roofs. First, high NDVI and low NIR values are used here to eliminate small vegetation and water objects. The other 3 parameters, i.e. pixel-based spectral class, morphological profiles and shape index, are integrated in a decision-making scheme to decide the likelihood of an object to be a building. All of them are rescaled to 1-9. The shape index plays some form of quantitative measure, i.e. building is often a compact object, whereas the other two are more like qualitative measures. The higher the shape index value of an object is, the more likely it is a building roof. Now, the operator needs to make the final decision in this multi-criteria evaluation scheme.

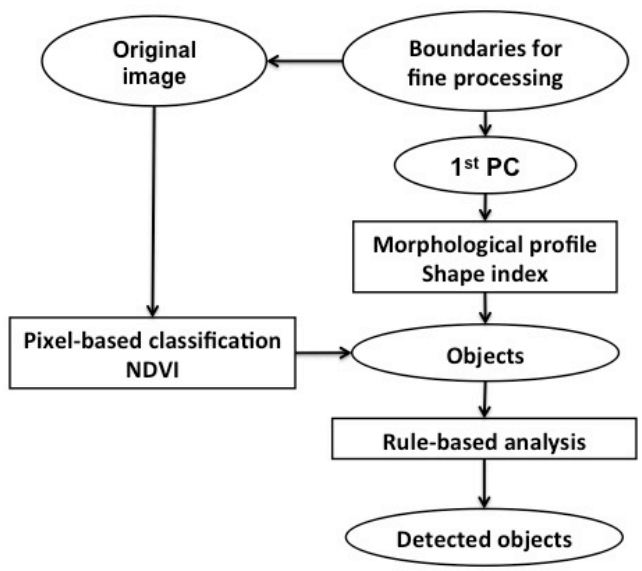

Figure 2. Fine level processing

The fully automatic processing can produce the detected objects using some predefined thresholds and rules. However, to ensure a good accuracy, the final step is designed for user's decision following the experiences that it is still difficult to detect the damage with current high-resolution satellite images (Ehrlich et al. 2009, Vu and Ban 2010). This is also the main reason why the rule-based analysis is chosen in development, which allows the users to input their knowledge to control the process. An automated classification, even adopting some complex machine learning algorithms, still cannot be reliable if the data has their own limitation.

\subsection{Design for parallel implementation}

As breaking into 2 levels, a big object formed on coarse level play as the tertiary to focus fine level processing within its boundary. The first goal of this design is not to analyse in details a homogeneous big object and hence, to speed up the processing. More importantly, the idea behind is to allow a parallel way of implementation in which the piece of information within each heterogeneous object will be delegated to a separate CPU. By this way, both data- and task- based implementation would be achieved.

In addition, the most computational-time-consuming modules such as region merging and morphological profile will be implemented with MPI (Message Passing Interface) in line with implementing the tsunami damage estimation system
(Koshimura et al. 2010). A test on image of different sizes of those two modules is depicted in Figure 3. Computation time drastically increases when the image size is $1024 \times 1024$ pixels, especially with region merging. The test used a parameter of 32 for K-mean clustering prior to applying the SRM, which seems to be unnecessarily many. When an operator has some prior knowledge about the study area and ensures a limited number of land-cover classes, the number of classes can be reduced and so the computation time for region merging does.

As mentioned, the proposed solution is to assist the participants in the crowd to response to a catastrophe event. Thus, different implementation methods will be considered and deployed to suit the platform availability of various operators. The implementation aspect will be reported in next publication.

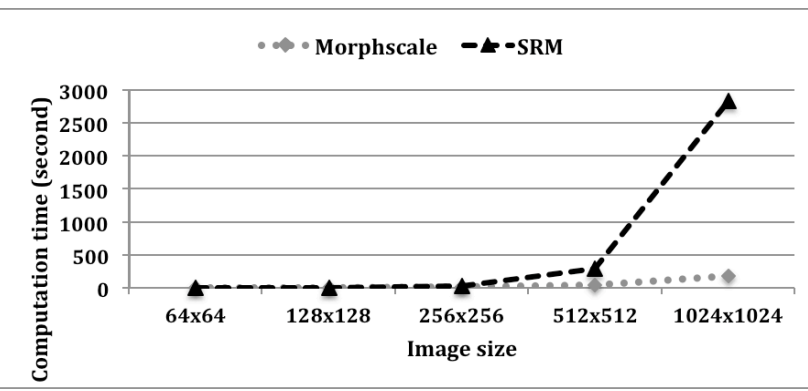

Figure 3. Computational time (Morphscale for morphological profile, SRM for region merging)

\section{RESULTS AND DISCUSSION}

Ban Nam Ken village, one of the most affected areas due to the 2004 Indian Ocean tsunami was selected as the study area with a QuickBird image captured on 2 January 2005, about a week after the tsunami attack. For demonstration of the developed solution, a portion of $1024 \times 1024$ was extracted, containing various surface types like vegetation, water, intact building roof, collapsed buildings and open soil, as shown in Figure 4a. The colour composite of brightness, greenness and homogeneous indices as the result of coarse level processing is illustrated in Figure $4 \mathrm{~b}$.

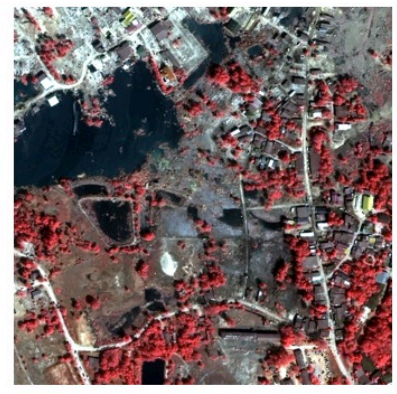

R: NIR, G: Red, B: Green (a)

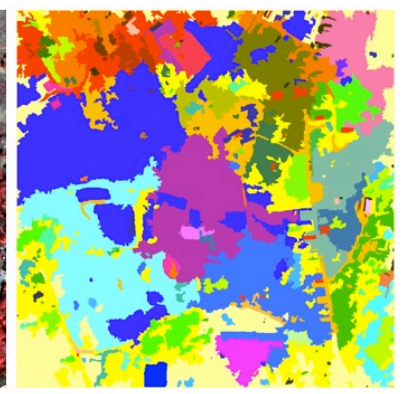

(b)
Figure 4. (a) False Colour Composite of original QuickBird image and (b) coarse level result

It is obvious that the bluish areas, i.e. low brightness, low greenness and high homogenous values, are the homogenous surface water areas and ignored in further fine processing. The greenness areas, however, needed to be reconfirmed with NDVI 
value to know whether it would be vegetation. The red to orange areas, which lack of blue homogeneity and green vegetation, is more likely the concrete covered areas and damage areas, if working on a post-disaster image. This is particularly true for the area at the top-left of our study area. In the case that a pre-event image was available, it would be able to detect the wash-away areas and possibly wet areas due to tsunami attack via change detection.

Figures 5 and 6 present the results of fine level processing of two selected areas, in which the extracted features presented in their identification numbers (ID) and their 'class'; the 'class' here is the combined results from pixel-based spectral, morphological, shape indices as a result of multi-criteria evaluation. The colour code for ID is just to discriminate the adjacent ones. Two clusters with the same colour but not next together do not have the same ID number. Figure 5 explores further the details of a damage area whereas Figure 6 presents the result from the non-damage area full of old rooftops.

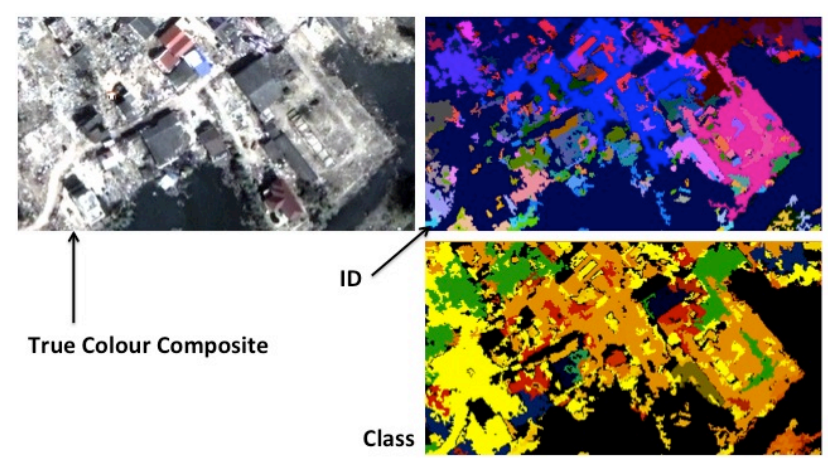

Figure 5. Fine level processing results of a damage area

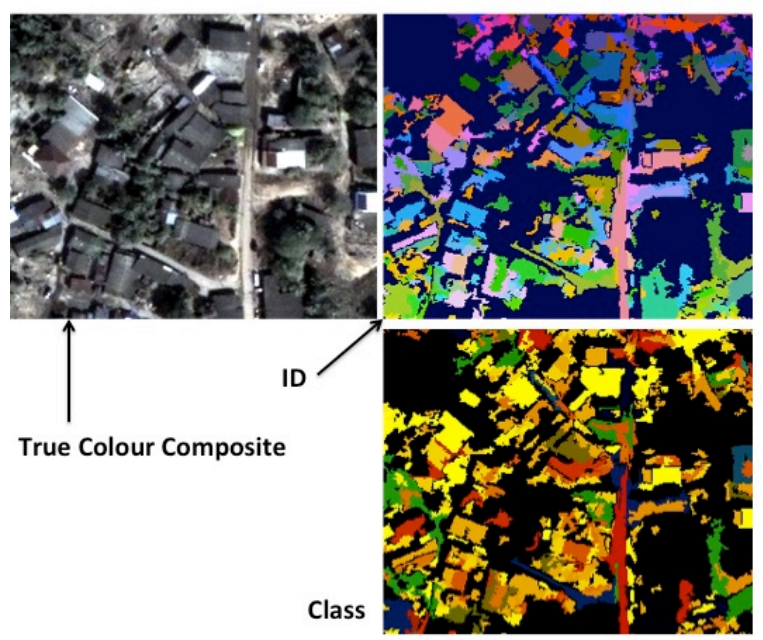

Figure 6. Fine level processing results of a non-damage area

Combining the evidences from both coarse and fine levels, a disaster-induced damage area can be confirmed. Current satellite spatial resolutions are unable to report detailed damage information at building level but only can delineate the noncollapse buildings and debris areas. The damage ratio is then computed approximately. Existing method prefers the pixelbased computation on a grid-based form, i.e. the ratio of number of collapsed pixels to total number of pixels in a cell. With the coarse level clusters by the developed solutions, the approximate damage ratio would be better and probably more easy to use in practice. One of the main reasons is that the street network is usually the boundary of administrative units and also clearly presented in satellite images, which lead the merging to follow.

Accuracy assessment of detected information from satellite image remains a big challenge though remote sensing images have been employed in disaster management for decades. It is mainly due to the gap between what remote sensing can produce and what the disaster management practitioners demands and get used to. There has been also a discussion on how damage information should be presented. Consequently, previous research (Gusella et al. 2005, Stramondo et al. 2006, Vu and Ban 2010) faced the difficulty in comparison with the 'ground truth' information.

In this paper, the detected buildings are simply crosschecked with the visually detected ones. It showed that as Figures 4,5 and 6 , the extracted results were reasonably matched with the reference ones. Most compact objects, more likely to be building rooftop, were well detected. Visually, the old house rooftops in the study area are not distinguishable from the surrounding implying that it would be tough for an automated processing as illustrated in Figure 7. The occlusion by the trees nearby also cleared a possible separation line between 2 objects introducing omission errors. More quantitative assessment will be reported in a mutual acceptable form with disaster management practitioners.

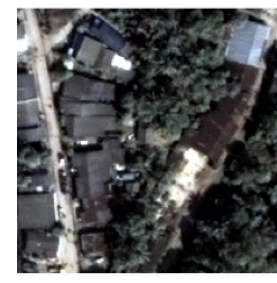

True Colour Composite

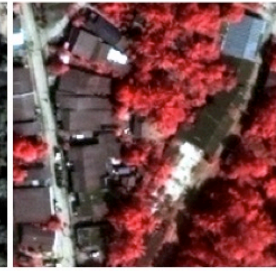

False Colour Composite

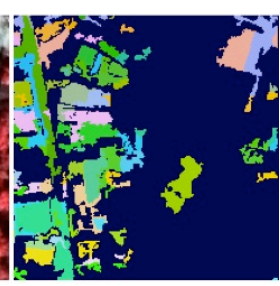

Figure 7. Difficult situation for automated recognition

\section{CONCLUSION}

Dual-scale processing framework has been introduced to support the rapid damage estimation at the early stage after a disaster. The initial development is to serve as part of a system for tsunami disaster damage estimation while its ultimate goal is to serve as early damage estimation solution for multi-type disaster in support of emergency responses and to distribute for detailed damage assessment. The test with QuickBird image of Ban Nam Ken, Phanga, Thailand produced a reasonably good result.

The result from coarse level delineated the highly suspected damage areas and produced the focused boundaries for fine level processing. The fine level processing designed as a semiautomatic approach then helps to explore the damage areas in further details and detect the non-collapsed buildings. The combination outcomes from both levels would enable the derivation of better damage ratio index. The solution was designed aiming at a parallel implementation, and detailed report of the computation time will be reported in next publication. However, to suit the available platform of various users, different way of implementation will be considered including multi-core CPU, GPU and grid platform. It is recommended to develop a suitable method for accuracy 
assessment dealing with objects and in the context of damage mapping.

\section{ACKNOWLEDGEMENT}

This study is a part of internationally collaborative research project supported by Industrial Technology Research Grant Program (Project ID: 08E52010a) from New Energy and Industrial Technology Development Organization (NEDO), Japan. The author acknowledges the financial support of the Faculty of Science conference grant, University of Nottingham, Malaysia campus.

\section{REFERENCES}

Adams, B.J., Huyck, C.K., Mansouri, B., Eguchi, R.T. and Shinozuka, M., 2004. Application of high-resolution optical Satellite imagery for post-earthquake damage assessment: The 2003 Boumerdeas (Algeria) and Bam (Iran) earthquakes. Multidisciplinary Center for Earthquake Engineering Research Progress and Accomplishments 2003-2004, University of Buffalo. http://mceer.buffalo.edu/publications/resaccom/04SP01/12_Eguchi.pdf (20 December 2007).

Balz, T. and Liao, M.S., 2010. Building damage detection using post-seismic high resolution SAR satellite data. International Journal of Remote Sensing, 31(13), pp. 3369-3391.

Bogaert, J., Rousseau, R., Hecke, P. V., and Impens, I., 2000. Alternative area-perimeter ratios for measurement of 2D shape compactness of habitats. Applied Mathematics and Computation, 111(1), pp. 71-85.

Bevington, J., Adams, B.J. and Eguchi, J., 2010. GEO-CAN Debuts to Map Haiti Damage. Imaging Notes, 25(2), http://www.imagingnotes.com/go/article.php?mp_id=208

Blaschke T., 2010. Object based image analysis for remote sensing. ISPRS Journal of Photogrammetry and Remote Sensing, 65, pp. 2-16.

Chesnel, A-L., Binet, R., and Wald, L., 2007. Quantitative Assessment of Building Damage in Urban Areas using Very High Resolution Images. In: Proceedings of Urban Remote Sensing 2007, 11-13 April 2007, Paris, France.

Ehrlich, D., Guo, H. D., Molch, K., Ma, J. W., Pesaresi, M., 2009. Identifying damage caused by the 2008 Wenchuan earthquake from VHR remote sensing data. International Journal of Digital Earth, 2, pp. 309-326.

Goodchild, M. F. and Glennon, J. A., 2010. Crowdsourcing geographic information for disaster response: a research frontier. International Journal of Digital Earth, 3, pp. 231-241.

Gusella, L., Adams, B. J., Bitelli, G., Huyck, C.K., \& Mognol, A., 2005. Object-oriented image understanding and postearthquake damage assessment for the 2003 Bam, Iran, earthquake. Earthquake Spectra, 21, pp. S225-S238.

Kerle, N., 2010. Satellite-based damage mapping following the 2006 Indonesia earthquake-How accurate was it? International Journal of Applied Earth Observation and Geoinformation, 12, pp. $466-476$.
Koshimura, S., Kayaba S., and Matsuoka M., 2010. Integrated approach to assess the impact of tsunami disaster. In: Safety, Reliability and Risk of Structures, Infrastructures and Engineering Systems, edited by Futura, Frangopol \& Sinnozuka, Taylor and Francis group, London.

Matsuoka, M. and Yamazaki, F., 1999. Characteristics of satellite images of damaged areas due to the 1995 Kobe Earthquake. In: Proceeding of the $2^{\text {nd }}$ Conference on the Applications of Remote Sensing and GIS for Disaster Management, The George Washington University, USA, CDROM.

Nock, R. and Nielsen, F., 2004. Statistical Region Merging. IEEE Transactions on Pattern Analysis and Machine Intelligence, 26(11), pp. 1452-1458.

Nock, R. and Nielsen, F., 2005. Semi-supervised statistical region refinement for color image segmentation. Pattern Recognition, 38, pp. 835-846.

Saito, K., Spence, R.J.S., Going, C. and Markus, M., 2004. Using high-resolution satellite images for post-earthquake building damage assessment: a study following the 26 January 2001 Gujarat Earthquake. Earthquake Spectra, 20, pp. 145-169.

Stramondo, S., Bignami, C., Chini, M., Pierdicca, N. and Tertullaiani, A., 2006. Satellite radar and optical remote sensing for earthquake damage detection: results from different case studies. International Journal of Remote Sensing, 27, pp. 44334447.

Vincent, L., 1993. Morphological grayscale reconstruction in image analysis: applications and efficient algorithms. IEEE Transactions on Image Processing, 2, pp. 176-201

Vu, T.T., Matsuoka, M., and Yamazaki, F., 2005. Detection and animation of damage using very high resolution satellite image following the 2003 Bam, Iran, earthquake. Earthquake Spectra, 21, pp. S319-S327.

Vu, T.T. and Ban, Y., 2010. Context-based damage mapping from high-resolution optical satellite images. International Journal of Remote Sensing, 31(13), pp. 3411-3425.

Yarbrough, L.D., Easson, G., and Kuszmaul, J.S., 2005. QuickBird 2 Tasselled Cap transform Coefficients: A comparison of derivation methods. In: Proceedings of Pecora 16 - Global Priorities in Land Remote Sensing, October 23-27, Sioux Falls, South Dakota, USA. 